\title{
Sexualidad en diversidad funcional intelectual: recopilación de los instrumentos de evaluación del equipo Salusex ${ }^{1}$
}

\section{Vicente Morell-Mengual}

Departamento de Psicología Evolutiva y de la Educación, Universitat de València, Valencia, España.

vicente.morell@uv.es

\section{Dolores Gil-Llario}

Departamento de Psicología Evolutiva y de la Educación, Universitat de València, Valencia, España.

dolores.gil@uv.es

\section{Irene Díaz-Rodríguez}

Departamento de Psicología Evolutiva y de la Educación, Universitat de València, Valencia, España.

irediazro@gmail.com

\section{Rafael Ballester-Arnal}

Departamento de Psicología Básica, Clínica y Psicobiología, Universitat Jaume I, Castellón de la Plana, España.

rballest@uji.es

\section{RESUMEN}

La sexualidad de las personas con discapacidad intelectual (DI) ha sido un tema muy controvertido. Su sexualidad ha estado sustentada por una serie de mitos y falsas creencias que, a pesar de carecer de apoyo empírico, han restringido su derecho a conseguir una expresión libre de esta faceta. En unos casos su sexualidad ha sido equiparada a la de los niños, creyendo que eran personas asexuadas; y en otros se les ha presupuesto una ausencia de control sobre sus impulsos, optando por no despertar sus instintos sexuales. El equipo SALUSEX inició el estudio de la sexualidad en este colectivo en el año 2015 con un proyecto financiado por el Ministerio de Economía y Competitividad relacionado con la calidad de vida y las secuelas del abuso sexual. Dadas las carencias y necesidades observadas, a este primer proyecto le siguió otro, actualmente en desarrollo, cuyo objetivo es la elaboración y el análisis de la eficacia de un programa de prevención del abuso y mejora de la salud sexual mediante el cual se están desarrollando y validando instrumentos psicométricos para recoger información sobre sus conocimientos, creencias y experiencias tanto de forma autoinformada como heteroinformada. Así, el presente trabajo tiene como propósito presentar algunos instrumentos para la evaluación de la sexualidad en el ámbito de la diversidad funcional intelectual desarrollados por el equipo Salusex.

Palabras clave: discapacidad intelectual; sexualidad; evaluación; cuestionarios; propiedades psicométricas 


\begin{abstract}
The sexuality of people with intellectual disabilities (ID) is a controversial issue. Their sexuality is characterised by myths and false beliefs that have restricted their right to achieve the experience of a free sexuality. In some cases, their sexuality was equated with that of children, as asexual people; and in other cases, a lack of control over their impulses was assumed, trying not to awaken their sexual instincts. In 2015, SALUSEX team initiated the study of sexuality in this group with a project, funded by the Ministry of Economy and Competitiveness, related to the quality of life and sequelae of sexual abuse. Taking into consideration the needs detected, this project was followed by another, currently under development, with the aim of developing and analysing the effectiveness of a programme to prevent abuse and improve sexual health. In this project, some psychometric instruments are being developed and validated to collect information about their knowledge, beliefs and experiences in both self-reported and other-reported. The aim of this paper is to present some instruments to assess the sexuality in the field of intellectual functional diversity, developed by Salusex team.
\end{abstract}

Key words: intellectual disability; sexuality; assessment; questionnaires; psychometric properties

${ }^{1}$ Esta investigación ha sido realizada gracias a un proyecto de investigación financiado por el Programa Estatal de I+D+i de la Convocatoria 2018 Orientada a los Retos de la Sociedad del Ministerio de Ciencia, Innovación y Universidades (Proyecto RTI2018095538-B-I00). 


\section{INTRODUCCIÓN}

El colectivo de personas con discapacidad intelectual (DI) constituyen un subgrupo poblacional heterogéneo caracterizado principalmente por déficits en el funcionamiento adaptativo, que impiden la consecución de los estándares sociales y culturales para la independencia personal y la responsabilidad social y limitan el funcionamiento en una o más actividades de la vida diaria; y por déficits en el funcionamiento intelectual, en solución de problemas, razonamiento, planificación, pensamiento abstracto, toma de decisiones, aprendizaje académico y aprendizajes a través de la propia experiencia (American Psychiatric Association, 2014). La concepción institucional que ha tenido este colectivo ha variado considerablemente según el periodo histórico y el área analizada, desde una mayor inclusión en relación a los aspectos laborales y ocupacionales (Olavarrieta et al., 2013) a una mayor contención en relación a aspectos relacionados con la sexualidad (Franco et al., 2012).

Durante un largo período, la sexualidad de las personas con DI se sustentó sobre algunos mitos y falsas creencias que, sin contar con evidencia científica, impidieron o dificultaron la expresión de su sexualidad (Tamas et al., 2019). Existían posturas totalmente polarizadas que establecían desde una ausencia total de deseo sexual, presuponiéndoles una asexualidad intrínseca o una conducta sexual libidinosa e incontable, presuponiéndoles una total ausencia del control de sus impulsos (Bazzo et al., 2007; Borawska-Charko et al., 2017). No obstante, en los últimos años la Convención de las Naciones Unidas sobre los Derechos de las Personas con Discapacidad ha establecido que este colectivo tiene el mismo derecho que el resto de la población a derecho a gozar del mayor nivel de salud posible, incluso en el ámbito de la salud sexual y reproductiva, sin discriminación. Así, progresivamente se han ido adoptando posturas derivadas del Modelo multidimensional del funcionamiento humano (AAIDD, 2010), centradas en la provisión de los apoyos necesarios.

Esta progresiva normalización ha propiciado un creciente interés por el estudio de las necesidades sexuales de este colectivo, siendo para ello necesario la elaboración y validación de instrumentos psicométricos adaptados a sus características que permitan obtener información sobre las áreas de intervención prioritarias. Existen algunos instrumentos como, por ejemplo, la Sexual Knowledge, Attitudes, Experiences, and Needs Scale (Siebelink et al., 2006) o la Sexual abuse prevention knowledge scale (Liou, 2014), que no han sido validados en nuestro contexto. Otros han sido desarrollados desde una perspectiva cualitativa a través de entrevistas o grupos focales y no aportan información suficientemente objetiva (Löfgren-Mårtenson et al., 2015). Otra gran limitación es idiosincrática de las limitaciones en el funcionamiento intelectual y en las pobres habilidades lingüísticas que presentan algunas personas con DI (American Psychiatric Association, 2014). Por este motivo algunas personas son incapaces de reflexionar sobre su propias vivencias o sentimientos. Por tanto, también es necesario recoger información desde la perspectiva de los profesionales o de los padres ya que esta información está menos sesgada y tiene mayores garantías de veracidad (Gil-Llario, Morell-Mengual et al., 2019).

En este sentido, y tenido en consideración las limitaciones enumeradas, el grupo SALUSEX inició el estudio de la sexualidad en este colectivo hace casi una década obteniendo financiación a través de proyectos $\mathrm{I}+\mathrm{D}+\mathrm{i}$ en las convocatorias de Retos para la Sociedad del Ministerio de Economía y Competitividad en 2015, y del Ministerio de Ciencia e Innovación en 2018. El objeto de estudio en un primer momento fue el análisis de las necesidades y características de la sexualidad de las personas con diversidad funcional intelectual, atendiendo en especial al modo en que éstas afectan a su calidad de vida, así como las dimensiones del abuso sexual atendiendo tanto a la prevalencia como 
las secuelas del mismo. Seguidamente se desarrolló, una vez identificadas las principales carencias y demandas, un programa de educación afectivo-sexual que en estos momentos es objeto de análisis en cuanto a su eficacia para la mejora de la salud sexual y la prevención del abuso proporcionándoles estrategias de identificación de indicios de riesgo, así como mecanismos de defensa ante dichos abusos.

Para poder identificar las necesidades de forma objetiva, válida y fiable, en una primera fase, y para testar las posibles mejoras derivadas de la aplicación del programa, en un segundo momento, el equipo Salusex, dada la ausencia de materiales de evaluación que cumplieran los criterios de fiabilidad y validez exigidos se propuso (1) la elaboración y validación de instrumentos psicométricos específicos que permitieran recoger información sobre sus conocimientos, creencias y experiencias tanto de forma autoinformada como heteroinformada. Es decir, se consideró crear instrumentos paralelos que pudieran ser cumplimentados por padres y profesionales con objeto de analizar el nivel de congruencia o discrepancia entre las distintas fuentes. Por otra parte, tras haber diseñado e implementado un programa de educación afectivo-sexual especialmente adaptado a las características identificadas, se propuso (2) la creación de instrumentos de evaluación de la eficacia del mismo. Todas estas aportaciones son fruto de un intenso trabajo realizado junto con diversos organismos públicos. Esta estrecha colaboración permite tener un contacto directo con la realidad psicosocial, además de favorecer la transferencia. Así, el presente trabajo tiene como propósito presentar algunos trabajos de construcción y validación de instrumentos para la evaluación de la sexualidad desarrollados recientemente por el equipo Salusex en el ámbito de la diversidad funcional intelectual.

\section{MÉTODO}

\section{Participantes}

En el primer estudio participaron 180 hombres y 180 mujeres con diferente grado de DI beneficiarias de algún tipo de recurso asistencial. Respecto a la edad, casi la mitad de la muestra tenía entre 45 y 55 años (45.8\%), mientras que el $28.3 \%$ tiene entre 31 y 44 años y el $25.8 \%$ entre 18 y 30 años. Respecto al nivel de discapacidad, el 58.9\% presentó una discapacidad intelectual leve y el $41.1 \%$ una discapacidad intelectual moderada.

En el segundo estudio participaron 246 personas con DI leve o moderada, el 55.3\% hombres y el $44.7 \%$ mujeres, con edades comprendidas entre los 19 y 64 años $(M=37.8$; DT $=10.43)$. La mayoría vivía con sus padres o tutores $(81.3 \%)$, seguidos por un $8.9 \%$ que vivía en residencias y un $6.9 \%$ en viviendas tuteladas con diferentes grados de supervisión.

En el tercer estudio participaron 65 padres y 53 madres de adultos con discapacidad intelectual que asisten a 15 Centros Ocupacionales situados en diferentes localidades de la provincia de Valencia y pertenecen a la Asociación Coordinadora de Centros Ocupacionales de la Comunidad Valenciana (COPAVA).

En el cuarto estudio participaron 100 profesionales de 20 centros ocupacionales ubicados en distintas localidades de España. Cada profesional evaluó a 2-3 usuarios de su centro completando un total de 246 cuestionarios. La muestra de usuarios estaba conformada por 136 hombres y 110 mujeres con DI leve. El rango de edad estaba comprendido entre los 18 y los 65 años $(M=37.58$; DT = 10.44). En cuanto a su lugar de residencia, el $81.3 \%$ vivía con familiares, el $8.9 \%$ vivía en una residencia u hospital, el $6.9 \%$ vivía en un entorno comunitario y el $2.8 \%$ vivía de forma independiente. 
En el quinto estudio participaron 625 mujeres y 478 hombres. El 68.5\% eran personas de la población general, el $16 \%$ eran padres o familiares de personas con DI y el $15.5 \%$ eran profesionales que trabajaban con personas con DI. La distribución de los participantes por edad fue: $46.3 \%$ entre 20 y 35 años; $26.8 \%$ entre 36 y 51 años; $20.9 \%$ entre 52 y 66 años y $6 \%$ entre 67 y 92 años. En cuanto al nivel educativo, la mayoría poseía estudios universitarios (42.2\%), el 30.9\% tenía estudios secundarios, el $23.3 \%$ tenía estudios primarios y solo una minoría no tenía estudios (3.7\%).

\section{Procedimiento y análisis de datos}

Para determinar las propiedades psicométricas de los distintos instrumentos en todos los estudios de validación se realizaron los siguientes análisis:

1. Prueba de Kaiser-Meyer-Olkin y la prueba de esfericidad de Bartlett como paso previo para para determinar las propiedades de la matriz de correlación de los ítems.

2. Análisis factorial exploratorio (AFE) y análisis factorial confirmatorio (AFC). El análisis de la bondad del ajuste del modelo factorial se realizó con los siguientes índices: Chi-cuadrado (X2), Chi-cuadrado relativo (X2/df), significación general del modelo (p), error cuadrático medio de aproximación (RMSEA), e índices de ajuste (CFI e TLI). Se consideró un ajuste apropiado cuando el valor de X2 era significativo ( $\mathrm{p}<.05$ ), el valor de $\mathrm{X} 2 / \mathrm{df}$ se situaba entre 1 y 2 , el CFI y el IFI eran ב.95, y el RMSEA $\leq .05$ (Bagozzi y Yi, 2011).

3. Exploración de la invarianza factorial mediante la realización de un AFC multigrupo para asegurar que diferentes aspectos de la estructura factorial son similares en función del género u otras variables.

4. Evaluación de su consistencia interna en términos de correlaciones ítem-escala y alfa de Cronbach.

5. Validez convergente en términos del coeficiente de correlación de Pearson entre las puntuaciones generales (y los factores) de cada escala y otros instrumentos utilizadas para la evaluación de constructos similares.

\section{RESULTADOS}

\section{Instrumentos autoinformados}

CUSEXDI. Evaluación de la sexualidad de las personas con discapacidad intelectual (Gil-Llario, Ballester-Arnal, Fernández-García et al., 2020). El CUSEXDI es una entrevista semiestructurada conformada por 34 ítems, 26 de respuesta cerrada y 8 de respuesta abierta, que recoge información sobre diversos aspectos relacionados con la sexualidad de las personas con DI. Incluye cuestiones relacionadas con los comportamientos sexuales, la conducta preventiva, la información sobre sexualidad recibida y las experiencias de abuso sexual. La fiabilidad, obtenida mediante el coeficiente Alfa de Cronbach reveló un valor de .73. En cuanto a la viabilidad, el instrumento fue valorado positivamente por parte de los profesionales, quienes consideraron que tanto las dimensiones como los ítems se ajustaban al perfil psicosocial de las personas con DI. A continuación, se describen los cuatro componentes.

- Comportamiento sexual: este apartado recoge información sobre el interés y atracción sexual hacia miembros de otro sexo o del mismo, las relaciones de pareja, 
las prácticas sexuales realizadas, la información sobre los cambios físicos percibidos en la pubertad y algunas vivencias relacionadas con la sexualidad, como las fantasías sexuales.

- Conducta preventiva: este componente evalúa los conocimientos y el uso de los diferentes métodos anticonceptivos.

- Formación recibida sobre sexualidad: este apartado evalúa el grado de información que se posee sobre sexualidad, haciendo hincapié en el tipo de información y quien la ha suministrado.

- Experiencias de abuso sexual: este último componente recoge información sobre las experiencias de abuso sexual y sus consecuencias psicosociales derivadas.

DRAS (DSARss). Escala de detección del riesgo de abuso sexual (Gil-Llario, Ballester-Arnal, Morell-Mengual et al., 2020). El DRAS es un cuestionario autoadministrado compuesto por 19 ítems que realiza un screening del riesgo de abuso sexual en personas con DI leve o moderada en cuatro dimensiones. El primer factor "aceptación del abuso por afecto" evalúa la incapacidad de identificar el abuso sexual cuando el agresor es un familiar, un cuidador y/o una persona por la que sienten afecto (por ejemplo, «mi padre me cuida, así que está bien tener relaciones sexuales con él»). El segundo factor "negación del riesgo asociado a lugares" valora la percepción del riesgo de abuso sexual en diferentes lugares públicos y privados (por ejemplo, «es imposible ser abusado sexualmente en mi casa»). El tercer factor "factores de riesgo y habilidades de autoprotección" evalúa diversas variables que incrementan o disminuyen la probabilidad de victimización, como el consumo de alcohol o el dominio de habilidades de afrontamiento (por ejemplo, «tengo más probabilidades de sufrir abusos sexuales si alguien me ofrece alcohol insistentemente»). El cuarto factor "desconocimiento de las reglas intimas" evalúa los conocimientos que la persona posee en relación con el espacio personal, la intimidad corporal y el contacto físico (por ejemplo, «está bien si alguien que conozco me toca el trasero»). El instrumento se contesta mediante una escala de respuesta dicotómica con las alternativas de «verdadero»y «falso». La puntuación total se calcula sumando un punto por cada respuesta incorrecta, obteniendo un valor que oscila entre 0 y 19. Una mayor puntuación indica un mayor riesgo de abuso sexual. El análisis factorial del DRAS reveló una estructura tetrafactorial que explicó el $65.34 \%$ de la varianza. La fiabilidad de la escala y de sus factores revela una buena consistencia interna tanto de la escala global $(\alpha=.82)$ como de sus cinco factores, aceptación del abuso por afecto $(\alpha=.93)$, negación del riesgo asociado a lugares $(\alpha=.73)$, factores de riesgo y habilidades de autoprotección $(\alpha=.81)$ y desconocimiento de las reglas intimas $(\alpha=.70)$. Además, la escala presenta una adecuada estabilidad temporal $(\mathrm{r}=.47)$ y una buena validez convergente, divergente y predictiva.

\section{Instrumentos heteroinformados}

ECS-PA. Evaluación del comportamiento sexual de personas con discapacidad intelectual para padres (Gil-Llario, Elipe-Miravet et al., 2019). Constituye un instrumento heteroinformado de 32 ítems dirigido a padres y madres de personas con discapacidad intelectual que evalúa diversos aspectos relacionados con el comportamiento sexual. Los tres factores que conforman la escala son: comprensión de la privacidad y normas sociales (PRIV-NOR); percepción de los conocimientos sobre sexualidad (CONOSEX); reocupación por un comportamiento sexual desinhibido (COMP-DESINH). El primer factor "PRIV-NOR" evalúa la comprensión de las normas sociales que los padres creen que su hijo o hija posee en relación a lo que es correcto hacer en público o en 
privado y en modo en que se aprendieron dichas normas (por ejemplo, «¿Entiende su hijo/a otras normas sociales acerca de la privacidad?» o «su hijola busca privacidad cuando se desnuda»). El segundo factor "CONO-SEX" evalúa el nivel de conocimientos que los padres creen que su hijo o hija posee en reacción al proceso reproductivo, los anticonceptivos, las ITS y los distintos tipos de relaciones (por ejemplo, «icree que su hijola sabe qué son los métodos anticonceptivos?» o « ¿se beneficiaría su hijola de una adecuada educación sexual?»). El tercer factor "COMP-DESINH" recoge las preocupaciones que tienen los padres de que su hijo o hija tenga comportamientos inadecuados en público, tenga ideas erróneas sobre sexualidad o pueda ser víctima de abusos sexuales (por ejemplo, «¿ se ha masturbado alguna vez su hijo/a en público?» o «¿está preocupado porque su hijo/a tenga ideas equivocadas acerca del sexo?»). El cuestionario presenta un formato de respuesta dicotómico de «sí» y «no». Las puntuaciones en cada escala oscilan entre 0 y 13 en la escala PRIV-NOR; entre 0 y 10 en CONO-SEX; y entre 0 y 9 en COMPDESINH. Finalmente, el estudio de la fiabilidad revela una buena consistencia en las distintas escalas con valores alpha de Cronbach que oscilan entre .70 y .87.

ECS-PR (ASBKID). Evaluación del comportamiento y los conocimientos sexuales en personas con discapacidad intelectual (Gil-Llario, Morell-Mengual et al., 2020). Es un instrumento de 24 ítems para ser completado por los profesionales que trabajan con personas con DI en entornos ocupacionales que evalúa cinco aspectos de la sexualidad. El primer factor "preocupación por el comportamiento sexual inapropiado o desinhibido del usuario (BEH-UNINHIB)" evalúa algunas cuestiones relacionadas con la realización de conductas sexuales en público, los tocamientos inapropiados a otras personas y el hecho de hablar de la sexualidad de forma grosera o insistente (por ejemplo, « ¿se ha masturbado alguna vez en público?» o «¿es consciente de sus propias respuestas sexuales físicas?»). El segundo factor "percepción del conocimiento de los usuarios sobre la privacidad y las normas sociales (PRIV-NOR)" recoge elementos relacionados con el conocimiento de las normas sociales de privacidad e intimidad (por ejemplo, «es consciente de las normas sociales acerca de no dejar que otros toquen las zonas intimas de su сиеrрo»). El tercer factor "percepción de los conocimientos del usuario sobre sexualidad (KNOW-SEX)" evalúa diversos conocimientos relacionados con la higiene y la salud sexual, los diferentes tipos de relaciones, los comportamientos socialmente aceptables y el proceso de reproducción (por ejemplo, «¿piensa que tiene conocimientos sobre higiene sexual?» $\mathrm{o}$ «icree que entiende el concepto de método anticonceptivo?»). El cuarto factor "preocupación por la sexualidad del usuario (CONCERN)" recoge las preocupaciones que tienen los profesionales de que el usuario tenga comportamientos inadecuados, experimente soledad y corra el riesgo de sufrir abusos sexuales (por ejemplo, « ¿le preocupa que pueda ser víctima de abuso sexual?»o «¿está preocupado por si no encuentra un/a compañero/a sentimental?»). Se responde mediante una escala dicotómica de «sí» y «no». Las puntuaciones en las distintas escalas oscilan entre 0 y 5 en BEH-UNINHIB y PRIVNOR; entre 0 y 10 en KNOW-SEX; y entre 0 y 4 en CONCERN. Los análisis psicométricos confirman la bondad de estructuras tretrafactorial y revelan una buena fiabilidad en todas las subescalas que oscilan entre $\alpha=.74$ y 92 . Finalmente, el instrumento presenta una buena validez convergente analizada con otro instrumento análogo que evalúa aspectos similares desde la perspectiva paterna y materna.

\section{Instrumentos para la población general}

ADISEX (ASEXID). Evaluación de las Actitudes hacia la Sexualidad de las Personas con Discapacidad Intelectual (Gil-Llario, Fernández-García et al., 2021). El 
ASEXID es un cuestionario compuesto por 18 ítems que evalúa las actitudes hacia diferentes aspectos relacionados con la sexualidad de las personas con discapacidad intelectual (interés sexual, intimidad, capacidad de controlar los impulsos sexuales, etc.). Este instrumento se elaboró a partir de algunos ítems de la escala propuesta por Oliva y Calderón (2010) y se contesta mediante una escala tipo Likert de 5 puntos que indica su nivel de acuerdo/desacuerdo con cada afirmación: $1=$ muy en desacuerdo, $2=$ algo en desacuerdo, $3=$ ni de acuerdo ni en desacuerdo, $4=$ de acuerdo, $5=$ muy de acuerdo. El cuestionario presenta una estructura trifactorial compuesta por los siguientes factores: actitud normalizadora (NOR-A); actitud negativa (NEG-A); y actitud paternalista (PAT-A). $\mathrm{El}$ primer factor "NOR-A" recoge actitudes relacionadas con quienes piensan que la sexualidad de las personas con DI tiene las mismas características que la de las personas sin DI (por ejemplo, «es normal que las personas con DI vean pornografía»o «las personas con DI pueden tener una pareja»). El segundo factor "NEG-A" agrupa actitudes que consideran que las personas con DI tienen menos interés en la sexualidad, por lo que es innecesario e incluso peligroso hablar de este tema (por ejemplo, «hablar de sexo con personas con DI es animarles a practicarlo» o «la masturbación puede perjudicar a las personas con DI»). El tercer factor "PAT-A" evalúa la preocupación por la falta de control de la sexualidad de las personas con DI y los riesgos asociados a ella, como los embarazos no deseados, las ITS, etc. (por ejemplo, «las personas con DI pueden controlar sus impulsos sexuales» o «las personas con DI perciben el peligro del abuso sexual»). La puntuación total se calcula sumando cada ítem, obteniendo una puntuación mínima de 18 y una máxima de 90. Una mayor puntuación indica actitudes más proactivas hacia la sexualidad de las personas con discapacidad intelectual. La fiabilidad de la escala revela una adecuada consistencia interna en sus tres factores, actitud normalizadora $(\alpha=.86)$, actitud negativa $(\alpha=.71)$ y actitud paternalista $(\alpha=.66)$. y una buena validez convergente.

\section{CONCLUSIONES}

El objetivo de este estudio ha sido presentar las propiedades psicométricas de cinco instrumentos dirigidos a la evaluación de la sexualidad en personas con discapacidad intelectual: dos autoinformados, el CUSEXDI y el DRAS; dos heteroinformados, uno para padres y madres, el ECS-PA, y otro para profesionales, el ECS-PR; y un instrumento para la evaluación de las actitudes hacia la sexualidad de las personas con DI para la población general, el ADISEX.

El CUSEXDI constituye un instrumento psicométricamente útil tanto para obtener información sobre los comportamientos sexuales de las personas con DI, como para comprobar de en qué medida son capaces estas personas de poner en práctica los conocimientos adquiridos. Los análisis realizados evidencian que el DRAS constituye un buen instrumento de cribado del riesgo de abuso sexual en personas con DI, ya que sus propiedades psicométricas avalan su fiabilidad y validez. Además, su brevedad y sencillez (apoyadas por ilustraciones) facilitan una evaluación rápida que permite obtener información valiosa para desarrollar programas preventivos. Cabe destacar que en breve van a salir publicados dos nuevos instrumentos de autoinforme: la versión para usuarios del ECS que se va a llamar SEBECOMID por sus siglas en inglés (Gil-Llario, et al., en revisión), y un instrumento de evaluación de los conocimientos especialmente útil para el análisis de la eficacia de cualquier programa de educación afectivo sexual denominado ISK-ID (Gil-Llario, Castro-Calvo et al., 2021).

El ECS-PA posee unas buenas propiedades psicométricas y proporciona una información muy útil de cara a la planificación y el diseño de intervenciones dirigidas a mejorar 
la salud sexual y centradas en los apoyos y necesidades personales. El ECS-PR constituye un instrumento válido y fiable para la evaluación del comportamiento y los conocimientos sexuales de las personas con DI. La información suministrada por esta herramienta puede orientar a los distintos profesionales que trabajan en centros ocupacionales a mejorar la información sobre la salud sexual e identificar las áreas que necesitan ser abordadas en el diseño de intervenciones que impliquen un apoyo individualizado. Finalmente, el ADISEX constituye un instrumento válido, fiable y útil tanto en la identificación de las ideas negativas hacia la sexualidad de las personas con DI, como para la concienciación sobre la necesidad de educar a la sociedad sobre la importancia de una educación afectivo-sexual integral y adaptada a las características distintivas de este colectivo. La calidad y el tipo de educación que reciben estas personas depende de las actitudes que poseen sus cuidadores y figuras de referencia.

La labor realizada por el equipo Salusex contribuye a llenar un importante vacío existente en la evaluación auto y heteroinformada de la salud sexual de las personas con diversidad funcional intelectual.

\section{REFERENCIAS}

AAIDD (2011). Discapacidad Intelectual. Definición, Clasificación y Sistemas de Apoyo Social. Alianza Editorial.

American Psychiatric Association. (2014). DSM-5. manual diagnóstico y estadístico de los trastornos mentales. Editorial Medica Panamericana.

Bagozzi, R. P., \& Yi, Y. (2011). Specification, evaluation, and interpretation of structural equation models. Journal of the Academy of Marketing Science, 40(1), 8-34. http://doi.org/10.1007/s11747-011-0278-X

Bazzo, G., Nota, L., Soresi, S., Ferrari, L., \& Minnes, P. (2007). Attitudes of Social Service Providers towards the Sexuality of Individuals with Intellectual Disability. Journal of Applied Research in Intellectual Disabilities, 20(2), 110-115. https://doi.org/10.1111/j.1468-3148.2006.00308.x

Borawska-Charko, M., Rohleder, P., \& Finlay, W. M. L. (2017). The sexual health knowledge of people with intellectual disabilities: A review. Sexuality Research and Social Policy, 14(4), 393-409. https://doi.org/10.1007/s13178-016-0267-4

Franco, D. G., Cardoso, J., \& Neto, I. (2012). Attitudes towards affectivity and sexuality of people with intellectual disability. Sexuality and Disability, 30(3), 261-287. https://doi.org/10.1007/s11195-012-9260-X

Gil-1lario, M. D, Elipe-Miravet, M., Giménez-García, C., Bisquert-Bover, M. y CastroCalvo, J. (2019). Validación de un instrumento de evaluación del comportamiento sexual de personas con discapacidad intelectual para padres (ECS-PA). International Journal of Developmental and Educational Psychology, 1(4), 519-528. https://doi.org/10.17060/ijodaep.2019.n1.v5.1634

Gil-Llario, M. D., Ballester-Arnal, R., Fernández-García, O. y Morell-Mengual, V. (2020). La evaluación de la sexualidad de las personas con discapacidad intelectual: construcción y descripción de una entrevista semiestructurada. International Journal of Developmental and Educational Psychology, 1(1), 379-392. https://doi.org/10.17060/ijodaep.2020.n1.v1.1795 
Gil-Llario, M. D., Ballester-Arnal, R., Morell-Mengual, V., Caballero-Gascón, L. y Castro-Calvo, J. (2020). Development and psychometric properties of the detection of sexual abuse risk screening scale (DSARss). Sexual Abuse 32(7), 850-877. https://doi.org/10.1177\%2F1079063219858061

Gil-Llario, M. D., Castro-Calvo, J., Fernández-García, O., Elipe-Miravet, M. y BallesterArnal, R. (2021). Estimating sexual knowledge of people with mild intellectual disability through a valid and reliable assessment scale: the ISK-ID. Journal of Applied Research in Intellectual Disabilities. https://doi.org/10.1111/jar.12909

Gil-Llario, M. D., Flores-Buils, R., Elipe-Miravet, M., Fernández-García, O. y BallesterArnal, R. (en revisión). Development and psychometric properties of a self-reported instrument for the Assessment of Sexual Behaviour and Concerns of people with Mild Intellectual Disabilities (SEBECOMID-S) Journal of Applied Research in Intellectual Disabilities.

Gil-Llario, M. D., Morell-Mengual, V., Díaz-Rodríguez, I. y Ballester-Arnal, R. (2019). Prevalence and sequelae of self-reported and other-reported sexual abuse in adults with intellectual disability. Journal of Intellectual Disability Research, 63(2), 138148. https://doi.org/10.1111/jir.12555

Gil-Llario, M. D., Morell-Mengual, V., Fernández-García, O., Castro-Calvo, J. y Ballester-Arnal, R. (2020). Development and psychometric properties of an instrument for the Assessment of Sexual Behaviour and Knowledge of people with Intellectual Disability. Journal of Applied Research in Intellectual Disabilities. https://doi.org/10.1111/jar.12823

Gil-Llario, M. D, Fernández-García, O., Castro-Calvo, J., Caballero-Gascón y BallesterArnal, R. (2021). Validation of a Tool to Assess Attitudes Towards Sexuality of Individuals with Intellectual Disability (ASEXID): A Preliminary Study. Sexuality and Disability 39, 147-165. https://doi.org/10.1007/s11195-020-09650-3

Liou, W. (2014). An illustrated scale measuring the Sexual-Abuse prevention knowledge of female high school students with intellectual disabilities in taiwan. Sexuality and Disability, 32(2), 135-151. https://doi.org/10.1007/s11195-013-9312-x

Löfgren-Mårtenson, L., Sorbring, E., \& Molin, M. (2015). “T@ ngled up in blue”: Views of parents and professionals on internet use for sexual purposes among young people with intellectual disabilities. Sexuality and Disability, 33(4), 533-544. https://doi.org/10.1007/s11195-015-9415-7

Olavarrieta, S., Darín, L., Suárez, P., Tur, N., Besteiro, B., \& Gómez-Jarabo, G. (2013). Actitudes hacia la sexualidad, esterilización, maternidad/paternidad y habilidades de crianza de personas con discapacidad intelectual: Un estudio preliminar. Siglo Cero, 44(4), 55-69.

Oliva, L. \& Calderón, M. (2010). Elaboración de una escala de actitudes respecto a la sexualidad de personas con discapacidad mental. Revista Electrónica de Psicología Iztacala 13(4), 106-115.

Siebelink, E. M., de Jong, M. D., Taal, E., \& Roelvink, L. (2006). Sexuality and people with intellectual disabilities: Assessment of knowledge, attitudes, experiences, and needs. Mental Retardation, 44(4), 283-294. https://doi.org/10.1352/00476765(2006)44[283:SAPWID]2.0.CO;2 
Tamas, D., Jovanovic, N. B., Rajic, M., Ignjatovic, V. B., \& Prkosovacki, B. P. (2019) Professionals, Parents and the General Public: Attitudes Towards the Sexuality of Persons with Intellectual Disability. Sexuality and Disability 37, 245-258. https://doi.org/10.1007/s11195-018-09555-2 